\title{
Quantitative evaluation of bone marrow fat content and unsaturated fatty index in young male soccer players using proton magnetic resonance spectroscopy ( $\left.{ }^{1} \mathrm{H}-\mathrm{MRS}\right)$ : a preliminary study
}

\author{
Jian Wang ${ }^{1 \#}$, Peiwei Yi ${ }^{2,3 \#}$, Yaobin Huang ${ }^{1}$, Qinqin $\mathbf{Y u}^{4}$, Yingjie Mei ${ }^{5}$, Jialing Chen ${ }^{1}$, Yanqiu Feng ${ }^{2,3}$, \\ Xiaodong Zhang ${ }^{1}$ \\ ${ }^{1}$ Department of Medical Imaging, The Third Affiliated Hospital of Southern Medical University (Academy of Orthopedics Guangdong Province), \\ Guangzhou, China; ${ }^{2}$ School of Biomedical Engineering, Southern Medical University, Guangzhou, China; ${ }^{3}$ Guangdong Provincial Key Laboratory \\ of Medical Image Processing, Southern Medical University, Guangzhou, China; ${ }^{4}$ Department of Medical Imaging, Shanghai General Hospital, \\ Shanghai, China; ${ }^{5}$ China International Center, Philips Healthcare, Guangzhou, China
}

Contributions: (I) Conception and design: X Zhang, Y Feng; (II) Administrative support: X Zhang; (III) Provision of study materials or patients: Y Huang, Q Yu; (IV) Collection and assembly of data: P Yi, Y Mei, J Chen; (V) Data analysis and interpretation: J Wang, P Yi; (VI) Manuscript writing: All authors; (VII) Final approval of manuscript: All authors.

\#These authors contributed equally to this work.

Correspondence to: Yanqiu Feng, PhD. No. 1023-1063, Shatai South Road, Baiyun District, Guangzhou 510515, China. Email: foree@163.com; Xiaodong Zhang, MD, PhD. No. 183, Zhongshan West Avenue, Tianhe District, Guangzhou 510630, China. Email: ddautumn@126.com.

Background: Marrow fat exists as a distinct adipose tissue and plays a critical role in affecting both the quantity and quality of bone. However, the effect of soccer training on marrow fat has been rarely reported. This study aims to evaluate and characterize the marrow fat content and composition in different bone areas of soccer players and age-matched healthy subjects using proton magnetic resonance spectroscopy $\left({ }^{1} \mathrm{H}-\mathrm{MRS}\right)$. Methods: Between May 2020 and June 2020, 20 professional soccer players (20.7 \pm 0.9 years) and 20 agematched healthy subjects $(21.2 \pm 0.8$ years $)$ were enrolled in this cross-sectional study. The ${ }^{1} \mathrm{H}-\mathrm{MRS}$ were acquired from the $3^{\text {rd }}$ lumbar vertebrae, bilateral femoral necks, and distal tibias of all subjects using a singlevoxel point-resolved spatially localized spectroscopy (PRESS) sequence. Four soccer players underwent a second magnetic resonance (MR) examination within a 30-minute interval after the initial scan to evaluate test-retest reproducibility. Inter- and intra-observer measurement reliabilities were assessed using 10 randomly selected spectra from the soccer players group. All spectra were processed using the jMRUI software package (http://www.jmrui.eu/). Quantified water and lipid signals were used to calculate fat content (FC) and the unsaturated fatty index (UI).

Results: Compared with healthy subjects, we found that soccer players had a lower FC in L3 and bilateral femoral necks and higher UI in the left femoral neck $(\mathrm{P}<0.05)$. All FC and UI values of the bilateral distal tibias showed no significant differences between the two groups $(\mathrm{P}>0.05)$. The UI values of the right femoral neck or distal tibia were markedly higher than the left side in both inactive subjects and soccer players $(\mathrm{P}<0.05$, except for the femoral neck in players), and there were notable $\Delta \mathrm{UI}$ differences in the lower limbs between the soccer players and the healthy subjects $(\mathrm{P}<0.05)$.

Conclusions: Soccer practice can be considered a positive sport that contributes to decreasing FC in lumbar vertebrae and femoral necks and increasing the UI in femoral necks. Quantitative MRS provides an ideal modality to predict marrow fat metabolism caused by mechanical stimulation.

Keywords: Magnetic resonance spectroscopy (MRS); bone marrow; fat content (FC); unsaturated fatty index (UI); soccer players 
Submitted Jan 16, 2021. Accepted for publication May 08, 2021.

doi: 10.21037/qims-21-64

View this article at: http://dx.doi.org/10.21037/qims-21-64

\section{Introduction}

Bone mineral density (BMD) and marrow fat are two crucial biomarkers for bone-related studies (1). Adipocytes form marrow adipose tissue (MAT), and BMD is directly related to osteoblasts. Adipocytes and osteoblasts are closely related to each other because they are both differentiated from mesenchymal stem cells (MSCs) in bone marrow (2). Physical training is well recognized as a means of suppressing MAT depots and enhancing BMD (3). Soccer is one of the most popular sports worldwide and is characterized by multiple forms of exercises, including turns, jumps, and sprints, together with accelerations and decelerations (4). Previous studies have shown that soccer training provides positive effects on bone mineral content (BMC) and BMD, and presents higher BMC and BMD in weight-bearing sites, such as the lumbar spine, femoral neck, greater trochanter, and lower limbs, compared to sedentary controls (4-7). However, there are still no studies that have demonstrated the impact of soccer training on bone marrow fat.

Increasing evidence has suggested a complex relationship between MATs and bone health $(8,9)$. On the one hand, as a distinct fat depot different from white, brown, and beige adipocytes $(10,11)$, MATs release adipokines and free fatty acids, which interfere directly or indirectly with bone remodeling or hematopoietic function (11). On the other hand, unsaturated fatty acids can inhibit osteoclast differentiation and osteolytic activity, which is beneficial to bone shaping (12). Yeung et al. (13) observed that osteopenic and osteoporotic women presented increased marrow fat content (FC) and decreased unsaturated fat compared to young control subjects. Other studies on morbid obesity $(14,15)$, type 2 diabetes (16), fragility fracture (17), and osteoarthritis (18) also suggested that a variety of diseases are associated with an increase of marrow FC and/or decrease of unsaturated fatty acids. These findings suggest that MATs play a critical role in affecting bone quality.

In order to quantify MATs, chemical shift encoded imaging (CSE-MRI), and proton magnetic resonance spectroscopy ( $\left.{ }^{1} \mathrm{H}-\mathrm{MRS}\right)$ are two commonly used noninvasive methods. CSE-MRI methods, such as Dixon's technique, have achieved success in obtaining the distribution of FC. However, their robustness still needs to be greatly improved for the estimation of fat composition, owing to the used signal model and reconstruction approaches $(19,20)$. In comparison, ${ }^{1} \mathrm{H}-\mathrm{MRS}$ methods provide more detailed chemical shift information that enables a simultaneous and accurate quantitation of FC and composition (21). Thus, they possess a unique advantage in determining the role of marrow fat in normal physiology and pathology.

This study used the ${ }^{1} \mathrm{H}-\mathrm{MRS}$ technique to evaluate and characterize both the FC and unsaturated fatty index (UI) in the bone regions of $3^{\text {rd }}$ lumbar vertebrae, bilateral femoral necks, and distal tibias of professional male soccer players and age-matched inactive controls. The application of this quantitative magnetic resonance (MR) technique to assess the changes of marrow composition with soccer practice has been rarely reported to our knowledge. These results may help to understand the association between professional soccer training and changes in marrow fat.

\section{Methods}

The study was conducted following the Declaration of Helsinki (as revised in 2013) and was approved by the institutional review board of the Third Affiliated Hospital of Southern Medical University. Informed consent was obtained from all individual participants.

\section{Subjects}

This was a cross-sectional, case-control study. All professional soccer players regularly trained (5 to 6 times per week, 2 hours at a time) over at least 8 years. All players kicked the ball with their right foot. The regular training consisted of 20 min of warm-up and stretching exercises, 40-50 min of technical exercises (shooting, dribbling, passing, and ball control), plus running, plyometrics, and isometric exercises, 30-50 min of match practice, followed by another $10 \mathrm{~min}$ of stretching exercises. The healthy controls were untrained and had less than 3 hours of highpressure exercises per week.

The inclusion criteria were as follows: (I) males between 19 and 22 years of age; and (II) body mass index (BMI) 
less than $24.9 \mathrm{~kg} / \mathrm{m}^{2}$. Subjects were excluded if they met any of the following conditions: (I) had lesions that were accidentally identified by a musculoskeletal radiologist in the targeted regions during the conventional examination, such as bone marrow edema, neoplasm, or fracture; (II) had pre-existing bone diseases, such as tumors, metastases, and infectious disorders; (III) had familial or metabolic diseases (i.e., obesity, osteoporosis, and anorexia nervosa) that could affect BMD; (IV) previously used drugs that could affect bone metabolisms, such as rosiglitazone and pioglitazone; and $(\mathrm{V})$ had claustrophobia and could not keep still during the examination. All subjects underwent MR examinations at 10 am over 2 months between May and June 2020. Before the examination, the subjects were instructed to have a light breakfast (e.g., porridge, bread, or bun) and refrain from overeating and intensive training the day before the examinations. The following information was collected from recruited subjects: age, height, weight, smoking/ alcohol drinking history in the past two weeks, and exercise mode and frequency.

\section{${ }^{1} H$-MRS of bone marrow}

The MRS experiments were performed on a 3.0 Tesla whole-body MRI scanner (Ingenia, Phillips, Best, Netherlands). All subjects were examined in the supine position with a 16-channel posterior coil.

Axial and sagittal T1-weighted and fat suppression T2weighted MRI were first obtained for anatomical and morphological visual assessments and voxel positioning of the lumbar vertebrae, bilateral femoral necks, and bilateral distal tibias. T1- and T2-weighted MRI were performed using the following imaging parameters: T2-weighted spectral attenuated inversion recovery (SPAIR) sequence [repetition time/echo time $(\mathrm{TR} / \mathrm{TE})=3,072 \mathrm{~ms} / 80 \mathrm{~ms}$ with a $4 \mathrm{~mm} / 0.4 \mathrm{~mm}$ slice thickness/gap], and T1-weighted turbo spin-echo (TSE) sequence $(\mathrm{TR} / \mathrm{TE}=540 \mathrm{~ms} / 15 \mathrm{~ms}$, slice thickness $=4 \mathrm{~mm}$, gap $=0.4 \mathrm{~mm}$ ). The single-voxel point resolved spatially localized spectroscopy (PRESS) sequence without water suppression and outer volume saturation was implemented for ${ }^{1} \mathrm{H}-\mathrm{MRS}$ with the following parameters: TR/TE $=3,000 \mathrm{~ms} / 36 \mathrm{~ms}$, spectral width $=2,500 \mathrm{~Hz}$, and the number of data points $=2,048$. The number of signalaveraged (NSA) was 16 for the femoral neck, distal tibia, and vertebral spine. Five $15 \times 15 \times 15 \mathrm{~mm}^{3}$ volumes of interest (VOIs) were carefully placed within the bone marrow of the L3 vertebrae, bilateral femoral necks, and bilateral distal tibias, respectively (as depicted in Figure 1A,B,C).
After local shimming using the pencil beam (PB) volume method for each VOI to shim the static magnetic field $\left(\mathrm{B}_{0}\right)$, MRS was successively acquired from the five voxels mentioned above. To avoid motion artifact of the lumbar VOI due to breathing, subjects were required to breathe steadily during data acquisition. The VOIs were matched with the corresponding compartment to avoid inclusion of the surrounding cortical bone, leaving a $3-5 \mathrm{~mm}$ margin to avoid water contamination and resonances from outside the voxel due to chemical shift displacement artifact.

\section{Spectral fitting and data analysis}

All spectra were exported from the scanner and processed using a time-domain analysis with the jMRUI software package (http://www.jmrui.eu/) (22-24). The acquired signals were successively processed by the steps of zerofilling, exponential apodization, Fourier transformation, and phase correction. Spectral assignments were performed according to the reference from existing literature (25). For the spectra from the L3 vertebral body and femoral neck, there were four main peaks, including water peak at $4.7 \mathrm{ppm}$, methylene protons [-( $\left.\left.\mathrm{CH}_{2}\right) \mathrm{n}-\right]$ at $1.3 \mathrm{ppm}$, allylic methylene protons $\left(-\mathrm{CH}=\mathrm{CHCH}_{2}-\right)$ at $2.05 \mathrm{ppm}$, and mild olefinic protons $(-\mathrm{CH}=\mathrm{CH}-)$ at $5.3 \mathrm{ppm}$. Several L3 vertebral bodies with $5.3 \mathrm{ppm}$ were totally hidden under the wing of the water peak; however, olefinic protons could be resolved in all spectra from the femoral neck. For the spectra from the distal tibia, small lipid peaks like $0.9 \mathrm{ppm}\left(-\mathrm{CH}_{3}\right.$, methyl protons), $2.25 \mathrm{ppm}\left(-\mathrm{CH}_{2} \mathrm{CH}_{2} \mathrm{CO}-\right.$, $\alpha$-carboxyl protons), $2.75 \mathrm{ppm}\left(-\mathrm{CH}=\mathrm{CH}-\mathrm{CH}_{2}-\mathrm{CH}=\mathrm{CH}-\right.$, diacyl protons), and $4.2 \mathrm{ppm}\left(-\mathrm{CH}_{2}-\mathrm{O}-\mathrm{CO}-\right.$, glycerol protons) could also be identified in addition to the above four lipid peaks. For the spectra from the femoral neck, the identified peaks were superior to those of the L3 vertebral body but inferior to those of the distal tibia.

A widely used quantitative tool for MRS data, AMARES (advanced method for accurate, robust, and efficient spectral fitting) (26), was employed to fit the spectra. The starting values used in the non-linear least-squares fitting algorithm included a manually selected resonance frequency and the linewidth of each peak of interest. The water peak and main fat peak were freely varied within $\pm 0.05 \mathrm{ppm}$, while all fat peaks were constrained together with the distance between $1.3 \mathrm{ppm}$ fat peak and other fat peak constant. A Lorentzian model function was assumed for all peaks. All lipid peaks had a linewidth equal to that of the methylene peak at $1.3 \mathrm{ppm}$. The fitting results for one representative spectrum in the 

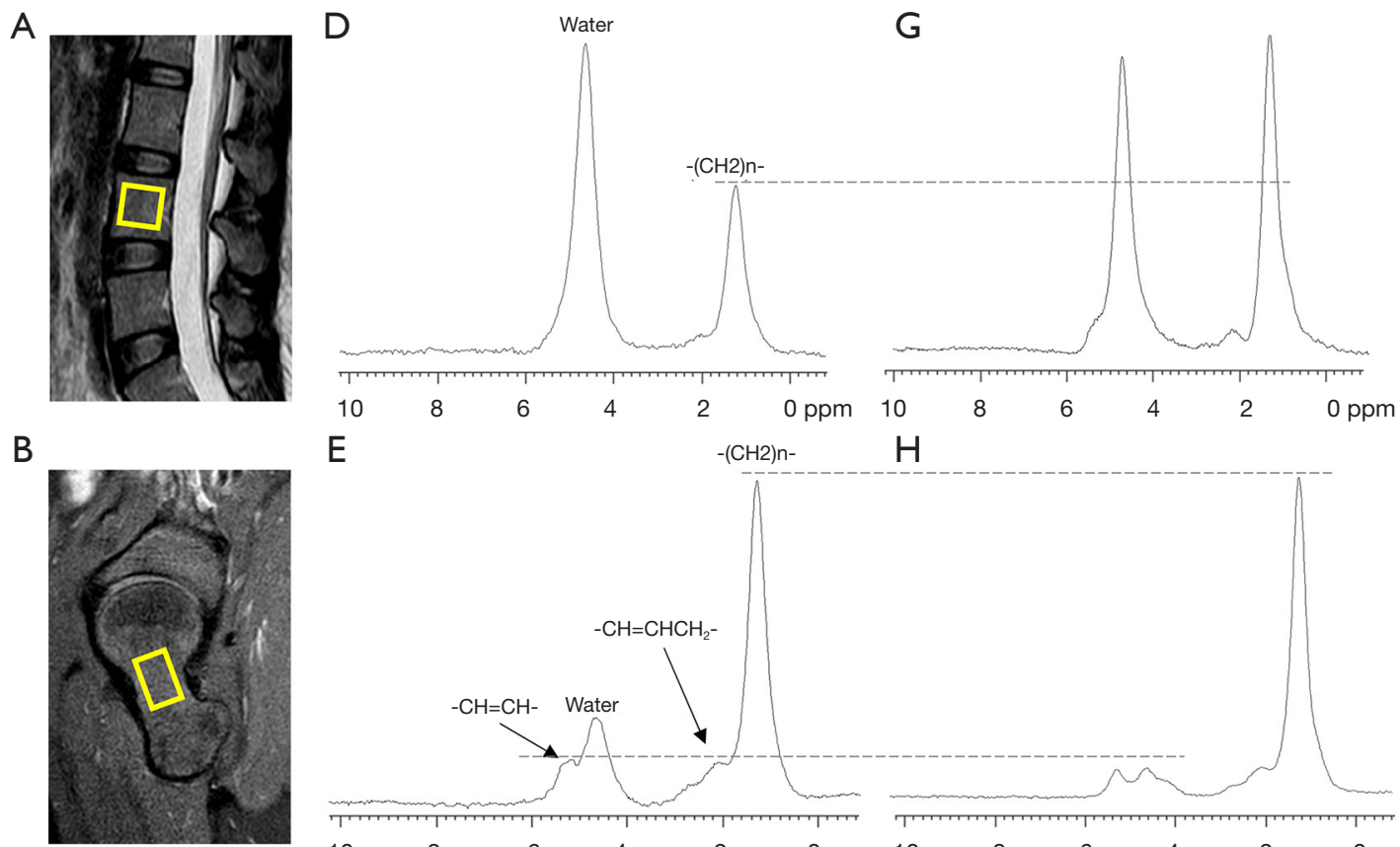

E

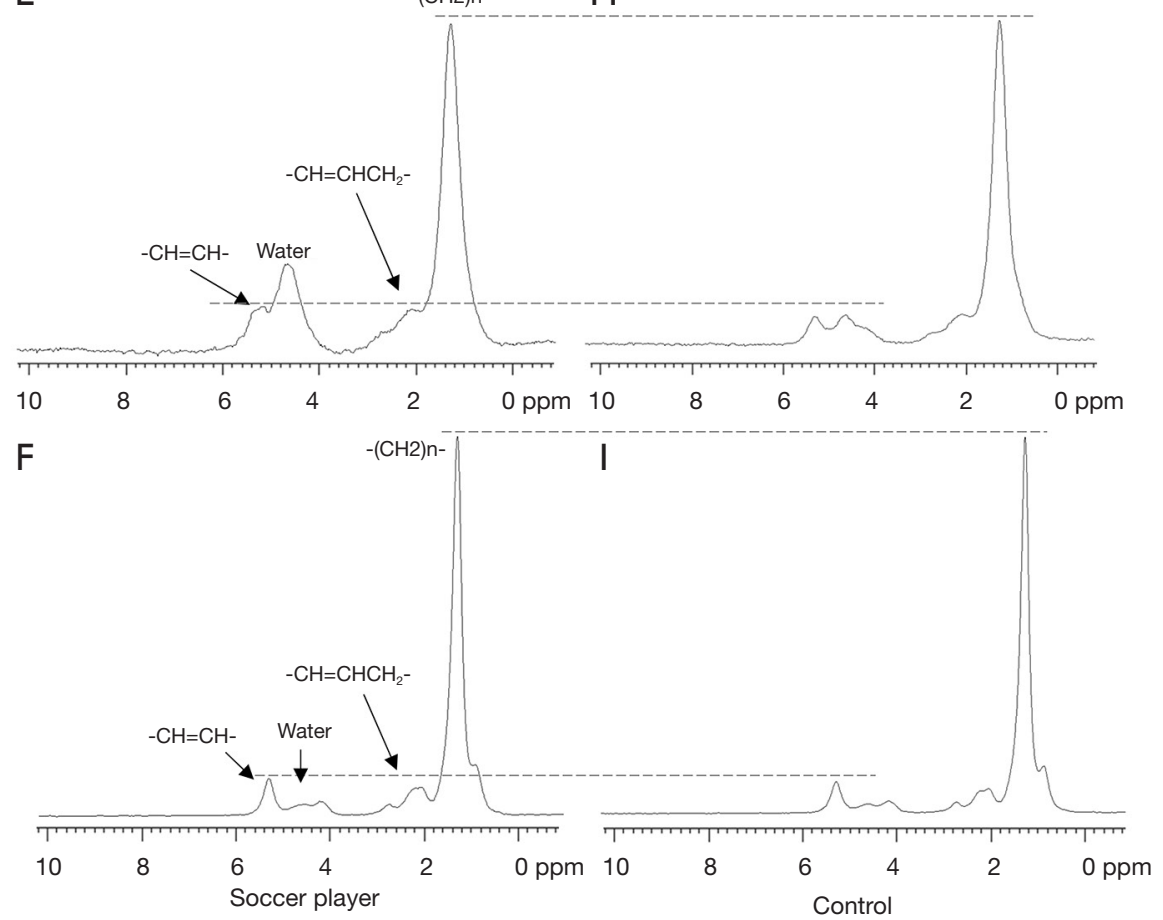

Figure 1 Voxel placement of the lumbar spine (A), femoral neck (B), and distal tibia (C). The ${ }^{1} \mathrm{H}-\mathrm{MRS}$ spectrum of the lumbar spine (D,G), femoral neck (E,H), and distal tibia (F,I) were combined fat peaks, like 1.3, 2.05, 5.3 ppm, and water peak. Small lipid peaks, like 0.9, 2.25, 2.75 , and $4.2 \mathrm{ppm}$, could also be identified, in addition to the above four peaks for the spectra of the distal tibia. The L3 centrum spectra (D,G) had relatively higher 4.7 ppm amplitude compared with the femoral neck (E,H) and distal tibia (F,I) in both male football players and agematched male healthy subjects, which indicates that the bone marrow composition of lumbar vertebrae is different from that of the lower limbs. Moreover, the players exhibited a relatively lower $1.3 \mathrm{ppm}$ in the lumbar spine (D) comparison of the controls (G). The 4.7 and 5.3 ppm amplitude of the femoral neck (E) of the soccer players were higher than that of the control subjects $(\mathrm{H})$. The spectra obtained for the distal tibia of the two groups exhibited similar lines (F,I). ${ }^{1} \mathrm{H}-\mathrm{MRS}$, proton magnetic resonance spectroscopy.

vertebral body, femoral neck, and distal tibia are shown in Figure S1A,B,C, respectively. Negligible residual signals could be found in all these spectra.

After spectral fitting, the amplitudes of the lipid and water peaks were quantified. FC was defined as the relative fat signal amplitude $(1.3 \mathrm{ppm})$ of the percentage of the total signal amplitude $(4.7$ and $1.3 \mathrm{ppm})$ and was calculated according to Eq. [1] $(13,27)$ :

$$
\text { Fat content }(\mathrm{FC} \%)=\frac{1.3 \mathrm{ppm}}{4.7 \mathrm{ppm}+1.3 \mathrm{ppm}} \times 100 \%
$$

UI was defined as the ratio of the unsaturated lipid at 
5.3 ppm to all lipid peaks \{Eq. [2]\} (16):

$$
\text { Unsaturated fatty index }(\mathrm{UI} \%)=\frac{5.3 \mathrm{ppm}}{1.3 \mathrm{ppm}+2.05 \mathrm{ppm}+5.3 \mathrm{ppm}} \times 100 \%
$$

$\Delta \mathrm{FC}$ and $\Delta \mathrm{UI}$ were defined as the $\mathrm{FC}$ and $\mathrm{UI}$ value deviation between the left and right femoral neck and distal tibia and were applied to compare the side-to-side differences. $\triangle \mathrm{FC}$ and $\Delta \mathrm{UI}$ were calculated to Eq. [3] and Eq. [4]:

$$
\begin{aligned}
& \Delta \mathrm{FC}=\mathrm{FC}(\text { Right limb })-\mathrm{FC}(\text { Left limb }) \\
& \Delta \mathrm{UI}=\mathrm{UI}(\text { Right limb })-\mathrm{UI}(\text { Left limb })
\end{aligned}
$$

In the vertebral body marrow, where the presence of a strong water signal and broadening of the spectral peaks caused the estimation of the olefinic peak challenging, only the FC value of the lumbar spine was calculated. However, both the FC and UI values were calculated to determine the femoral neck and distal tibia marrow fat composition. All of the FC and UI values were expressed as a percentage (\%).

\section{Statistical analysis}

Numerical data of all variables were reported as the mean \pm standard deviation (SD). Data were analyzed using SPSS (IBM, version 20.0), and the significance level was set at 0.05 . For MRS scanning agreement, Bland-Altman plots with $95 \%$ limits of agreement, intraclass correlation coefficients (ICC), as well as correlation and regression analyses were used for twice-examination repeatability on a group of players $(n=4)$ using repetitive scanning with a 30-minute interval after getting on and off the scanner. Reliability analysis was used to calculate the ICC and correlation coefficient for evaluating intra- and interobserver reliabilities by randomly selecting 10 spectra of a group of soccer players. Differences between the two groups were determined using independent samples $t$-tests. A paired $t$-test was performed to evaluate statistical differences between the left and right limbs for bone marrow parameters. Statistical difference analysis of FC and UI between the three regions (lumbar vertebrae, femoral necks, and distal tibias) was performed using one-way analysis of variance (ANOVA).

\section{Results}

\section{General characteristics}

Between May 2020 and June 2020, a total of 40 individuals were enrolled in this study (20 soccer players and 20 healthy subjects), with a mean age of $20.98 \pm 0.85$ years, and no significant differences between the two groups $(\mathrm{P}=0.058)$. The mean age of soccer players was $20.7 \pm 0.9$ years old, while that of the control group was $21.2 \pm 0.8$ years. The average height of the soccer players and controls were 179.2 \pm 6.1 and $176.6 \pm 3.8 \mathrm{~cm}$, respectively $(\mathrm{P}=0.154)$, while the average weight of the two groups were $69.6 \pm 6.1$ and $62.2 \pm 6.5 \mathrm{~kg}$, respectively $(\mathrm{P}=0.002)$. The mean $\mathrm{BMI}$ in the soccer player group was measured to be $21.7 \pm 1.2 \mathrm{~kg} / \mathrm{m}^{2}$, whereas in the healthy control group, this value was $19.9 \pm 1.7 \mathrm{~kg} / \mathrm{m}^{2}$, and this difference was statistically significant $(\mathrm{P}=0.001)$. None of the participants smoked or drank alcohol in the two weeks before the examination.

\section{Spectral characteristics of different anatomical sites and groups}

Good quality spectra were acquired from all subjects. Typical spectra consisted of water and several lipid peaks (as shown in Figure 1). Spectra obtained from the L3 of both groups had a relatively higher water content than the lower limbs. The femur and tibia marrow spectra exhibited a higher methylene peak amplitude than the lumbar marrow spectra, thus indicating that the lower limbs have a higher FC than that of the lumbar vertebrae. Compared to the healthy subjects, the soccer players had a relatively lower $1.3 \mathrm{ppm}$ amplitude in the lumbar vertebrae (Figure 1D,G) and a higher water peak in the femoral neck (Figure 1E,H). There was no statistical difference in the tibia marrow fat and water peak amplitude between soccer players and healthy controls (Figure 1F,I).

\section{Differences in FC and UI values between the soccer players and bealthy controls}

Compared with the healthy controls, the professional soccer players had a lower bone marrow $\mathrm{FC}$ in the $\mathrm{L} 3(39.59 \pm 12.67$ vs. $50.37 \pm 12.30, \mathrm{P}=0.010$ ) and bilateral femoral necks (left: $80.09 \pm 9.36$ vs. $88.59 \pm 5.71, \mathrm{P}=0.002$; right: $79.03 \pm 11.72$ vs. $87.34 \pm 6.21, \mathrm{P}=0.009)$, and a higher UI in the left femoral neck $(7.41 \pm 1.44$ vs. $6.33 \pm 1.63, \mathrm{P}=0.032)$. However, there was no notable difference in the UI value of the right femoral neck between the two groups $(\mathrm{P}>0.05)$. Moreover, the bone marrow FC and UI values of the bilateral distal tibias showed no differences between the soccer players and healthy subjects $(\mathrm{P}>0.05)$. All results are shown in Table 1 and Figure 2. 
Table 1 The mean \pm standard deviation of fat content and unsaturated fatty index values of L3, bilateral femoral necks, and distal tibias between the soccer players and control groups

\begin{tabular}{|c|c|c|c|c|c|c|}
\hline Location & \multicolumn{3}{|c|}{ Fat content (\%) } & \multicolumn{3}{|c|}{ Unsaturated fatty index (\%) } \\
\hline L3 & $39.59 \pm 12.67$ & $50.37 \pm 12.30$ & 0.010 & - & - & - \\
\hline Left femur & $80.09 \pm 9.36$ & $88.59 \pm 5.71$ & 0.002 & $7.41 \pm 1.44$ & $6.33 \pm 1.63$ & 0.032 \\
\hline Right femur & $79.03 \pm 11.72$ & $87.34 \pm 6.21$ & 0.009 & $7.87 \pm 1.35$ & $8.33 \pm 1.07$ & 0.249 \\
\hline Right tibia & $90.94 \pm 3.40$ & $91.73 \pm 2.71$ & 0.419 & $8.80 \pm 1.05$ & $8.63 \pm 1.16$ & 0.633 \\
\hline
\end{tabular}

Values are expressed as mean \pm standard deviation. ${ }^{a}$, calculated using the independent samples $t$-test to show the difference between soccer players and healthy subjects.
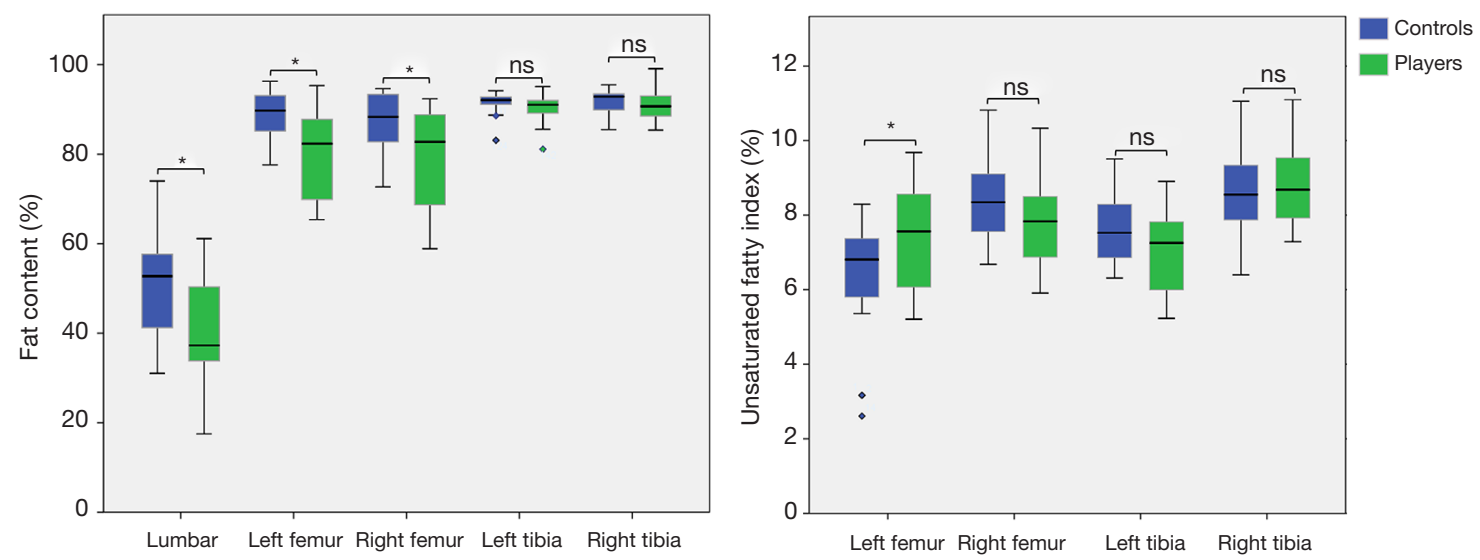

Figure 2 Boxplots of the bone marrow FC (left) and UI (right) values of the L3 vertebral body, bilateral femoral necks, and distal tibias in soccer players and healthy controls. The bone marrow FC of the L3 vertebral body and the femoral neck, and the bone marrow UI of the left femoral neck exhibited significant differences between the soccer players and control subjects. *, P<0.05; ns, P $\geq 0.05$. FC, fat content; UI, unsaturated fatty index; L3, $3^{\text {rd }}$ lumbar vertebrae.

\section{Differences in the FC and UI values among $3^{\text {rd }}$ lumbar vertebrae, bilateral femoral necks, and distal tibias}

All of the FC values between the left and right side of the femoral neck or distal tibia showed no marked differences between the two groups $(\mathrm{P}>0.05)$. However, the UI variable of the right femoral neck and distal tibia was significantly higher than the left side of the lower limbs in both groups $(\mathrm{P}<0.05$, except for the femoral neck in the soccer players group). Also, there were no statistically significant differences in the $\triangle \mathrm{FC}$ of the femoral neck and distal tibia between the players and the healthy controls (femur: $-1.07 \%$ vs. $-1.25 \%, \mathrm{P}=0.906$; tibia: $0.59 \%$ vs. $0.29 \%$, $\mathrm{P}=0.698)$. However, there were differences in the femoral neck and distal tibia $\Delta \mathrm{UI}$ between the two groups $(\mathrm{P}<0.05)$. All of the above data are displayed in Table 2 .

Different anatomical site FC value comparisons showed that there were significant differences between soccer players (L3 vs. femur vs. tibia: $39.59 \pm 12.67 v s .79 .89 \pm 10.61$ vs. $90.58 \pm 2.99)$ and healthy control (L3 vs. femur vs. tibia: $50.37 \pm 12.30 v s .87 .97 \pm 5.92 v s .91 .59 \pm 2.58)$. The FC of the distal tibia was higher than those of the L3 and femur in both groups. The mean UI values of the femur and tibia in healthy subjects were $7.33 \pm 1.70$ and $8.14 \pm 1.14$, respectively, with significant differences between the different sites of the lower extremity $(\mathrm{P}=0.035)$. Meanwhile, the UI in the soccer group showed no significant differences between the femoral neck and the distal tibia $(\mathrm{P}=0.392)$ (Table 3). 
Table 2 The mean \pm standard deviation of FC and UI values of the left and right femoral necks and distal tibias in the soccer players and control groups

\begin{tabular}{|c|c|c|c|c|}
\hline Variable & \multicolumn{2}{|c|}{ Fat content (\%) } & \multicolumn{2}{|c|}{ Unsaturated fatty index (\%) } \\
\hline \multicolumn{5}{|l|}{ Soccer players } \\
\hline Left & $80.09 \pm 9.35$ & $90.35 \pm 3.08$ & $7.41 \pm 1.44$ & $7.01 \pm 1.11$ \\
\hline Right & $79.03 \pm 11.72$ & $90.94 \pm 3.40$ & $7.87 \pm 1.35$ & $8.80 \pm 1.05$ \\
\hline \multicolumn{5}{|l|}{ Controls } \\
\hline Left & $88.59 \pm 5.71$ & $91.44 \pm 2.51$ & $6.33 \pm 1.63$ & $7.64 \pm 0.89$ \\
\hline Right & $87.34 \pm 6.21$ & $91.73 \pm 2.71$ & $8.33 \pm 1.07$ & $8.63 \pm 1.16$ \\
\hline$P$ value & 0.139 & 0.560 & 0.000 & 0.001 \\
\hline Controls & -1.25 & 0.29 & 2.00 & 0.99 \\
\hline $\mathrm{P}$ value & 0.906 & 0.698 & 0.008 & 0.049 \\
\hline
\end{tabular}

Values are expressed as mean \pm standard deviation. ${ }^{a}$, means difference value between the left and right lower extremity was calculated according to the equation: $\Delta=$ right - left. FC, fat content; UI, unsaturated fatty index.

Table 3 The mean \pm standard deviation of FC and UI values in L3, bilateral femoral necks, and distal tibias

\begin{tabular}{|c|c|c|c|c|c|c|c|}
\hline Groups & \multicolumn{4}{|c|}{ Fat content (\%) } & \multicolumn{3}{|c|}{ Unsaturated fatty index (\%) } \\
\hline Soccer players & $39.59 \pm 12.67$ & $79.89 \pm 10.61$ & $90.58 \pm 2.99$ & 0.000 & $7.64 \pm 1.40$ & $7.91 \pm 1.40$ & 0.392 \\
\hline Controls & $50.37 \pm 12.30$ & $87.97 \pm 5.92$ & $91.59 \pm 2.58$ & 0.000 & $7.33 \pm 1.70$ & $8.14 \pm 1.14$ & 0.035 \\
\hline
\end{tabular}

Values are expressed as mean \pm standard deviation. ${ }^{\text {a }}$, calculated using the one-way analysis of variance (ANOVA) test to demonstrate the difference among the L3 centrum, femoral neck, and distal tibia. FC, fat content; UI, unsaturated fatty index.

\section{Twice-examination repeatability, intra-, and inter- observer reproducibility}

Regression and Bland-Altman analyses using the MRSbased FC and UI measurements obtained from repeated scans are shown in Figure 3. The FC correlation was $\mathrm{r}=0.998$ and a $95 \%$ limit of agreement $(-2.289$ to 2.194$)$, and the UI correlation was $\mathrm{r}=0.955$ and a $95 \%$ limit of agreement ( -0.803 to 0.804$)$ between scans 1 and 2 . The ICCs of the FC and UI values were 0.999 and 0.995 , respectively, demonstrating good interscan reproducibility. The ICCs of the FC and UI intra-observer were 0.994 and 0.994 , respectively, and 0.950 and 0.947 for inter-observer. The intra- and inter-observer correlations were all $>0.950$, demonstrating good intra-observer and inter-observer reproducibility.

\section{Discussion}

In this study, we quantitatively assessed the bone marrow fat using ${ }^{1} \mathrm{H}-\mathrm{MRS}$ in young male professional soccer players and age-matched male healthy subjects. Compared with the healthy controls, the soccer players had decreased FC values in the lumbar spine and bilateral femoral necks but no significantly decreased $\mathrm{FC}$ values in the bilateral tibias. We also observed that there were side-to-side differences in the bone marrow UI values in the bilateral lower extremities in both groups (except for the UI values of femurs in soccer players). Compared with the healthy controls, the soccer players had a marked bone marrow side-to-side difference 

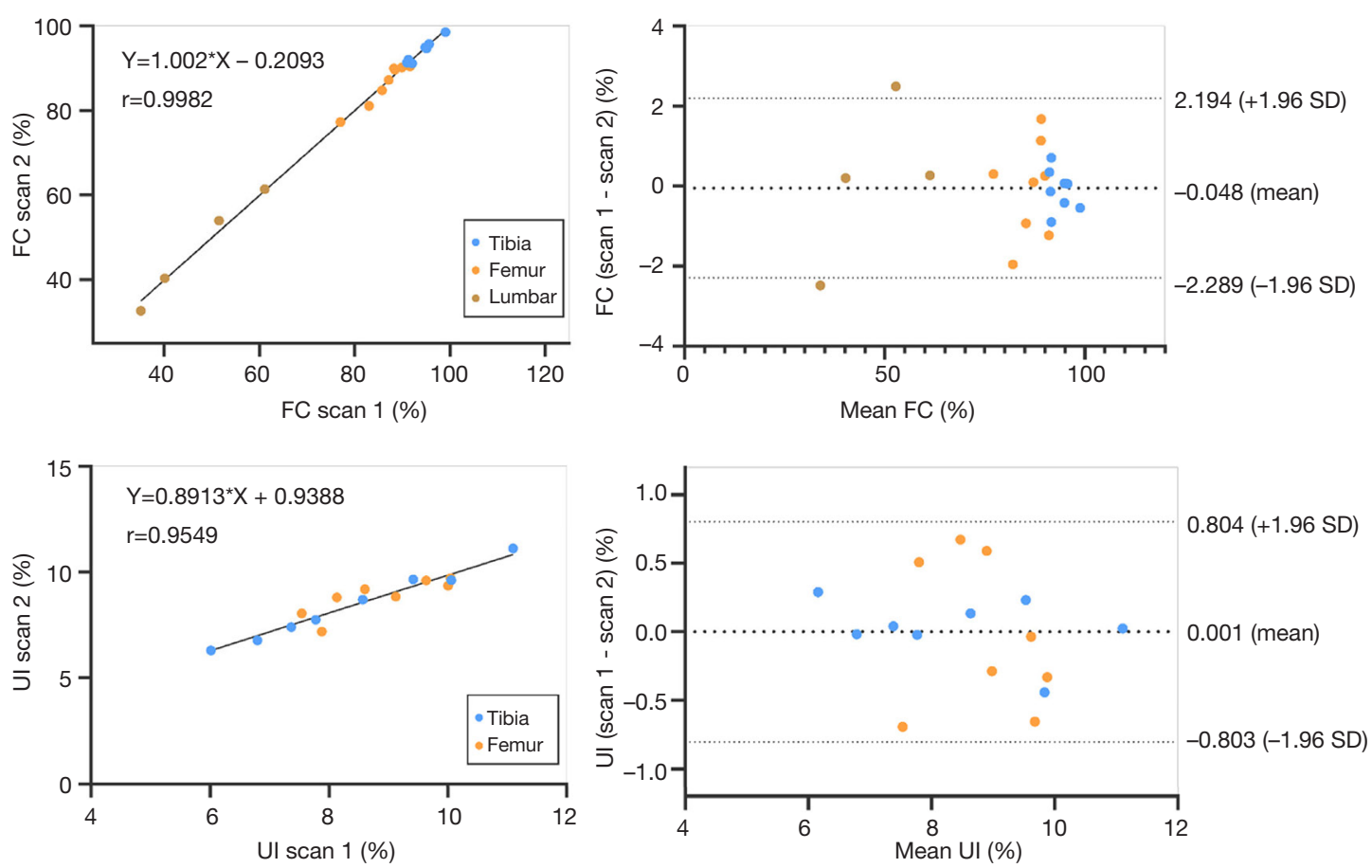

Figure 3 Scatter (left) and Bland-Altman (right) plots of FC (top row) and UI (bottom row) measurements based on 1H-MRS obtained from repeated MR scans. An excellent correlation, negligible bias, and $95 \%$ limitation of agreement of approximately $\pm 2.29 \%$ for FC and $\pm 0.81 \%$ for UI or less were demonstrated. FC and UI was expressed as a percentage of changes (\%). FC, fat content, UI, unsaturated fatty index, SD, standard deviation; $1 \mathrm{H}-\mathrm{MRS}$, proton magnetic resonance spectroscopy.

$(\Delta \mathrm{UI})$ in the femoral neck; however, this was unremarkable in the distal tibia. These findings revealed, for the first time, the characteristics of bone marrow fat in young male professional soccer players and the impact of soccer on bone marrow fat. The quantitative assessment of bone marrow using ${ }^{1} \mathrm{H}$-MRS may provide a new quantitative biomarker to study the relationship between soccer exercise and bone marrow.

In the present study, the spectrum of the L3 vertebrae had larger water peaks, and the spectra of the bilateral lower extremities had a relatively intense lipid peak from bulk methylene protons $(1.3 \mathrm{ppm})$ compared with the water peak, indicating higher water content on the spine and higher FC on the lower limbs. We also discovered that the tibia had the highest marrow FC values compared with the lumbar spine and femoral neck both in soccer players and sedentary controls, and the FC values of the bilateral distal tibias in the two groups were all above $90 \%$. These results are consistent with the findings of a previous study, which reported that the appendicular skeleton accumulated more adipocytes before the axial skeleton, and the distal ends of lower extremity bones were infiltrated first (8). The highest marrow FC on the appendicular skeleton might be a physiological phenomenon and may not be related to pathological change.

Our study also displayed lower FC values in the L3 vertebrae and bilateral femoral necks in elite soccer players than the age-matched sedentary controls, which suggests that soccer training could prevent fat accumulation in L3 and the femoral neck. High marrow FC has recently been proposed as an indicator of bone fragility $(28,29)$. Considering the higher bone marrow FC value assessed by ${ }^{1} \mathrm{H}-\mathrm{MRS}$, the present study may provide an indirect confirmation that soccer is associated with the improvement of bone health. Belavy et al. reported a decreased fat fraction in the lumbar vertebrae of runners as compared to their control subjects (who did not participate in regular sport in the last 5 years) using a 2-point Dixon MR sequence in vivo, which was similar to our finding of lower FC in the L3 vertebrae of soccer players (30). However, the mechanism through which sports affect bone marrow $\mathrm{FC}$ has not been elucidated. It may be explained from a mechanistic 
viewpoint, which holds that mechanical stress and loading inhibit adipocyte differentiation (31,32). Exercise succeeds in reducing the expression of peroxisome proliferatoractivated receptor-gamma (PPAR- $\gamma$ ) (33), which could down-regulate adipogenesis and contribute to lowering FC. Another explanation is "marrow reconversion" theory, where mechanical stimulation causes the reconversion from inactive yellow bone marrow to active red bone marrow (34-36), resulting in a decreased marrow FC and increased water content with soccer practice. Our study observed a decreasing tendency towards FC in the lumbar vertebrae and femoral neck, suggesting a positive influence of soccer practice stimulus on marrow FC.

The FC values of bilateral distal tibias in young male soccer players did not decrease compared to inactive healthy controls. The inconsistent changes in bone marrow $\mathrm{FC}$ values between different anatomical regions with soccer training may be related to the types of bone marrow adipose $(37,38)$. The MAT consists of two kinds of types: one is constitutive MAT (cMAT), which typically resides in the distal tibia, and the other is regulated MAT (rMAT), which is predominantly located in the proximal femur and lumbar spine $(11,38,39)$. The rMAT appears highly responsive to drive stimuli than cMAT, which may also contribute to the significant change in $\mathrm{FC}$ values at the femoral neck and lumbar spine compared with the distal tibia. It was worth noting that the lower marrow fat accumulation of the femoral neck and lumbar spine in soccer players may be consistent with the result of an in vivo study in which soccer players had higher BMC and BMD lumbar spine and femoral neck $(40,41)$.

Unlike the harmful effects of increased marrow fat on bone, Pino et al. (12) observed that unsaturated fatty acids have protective effects on bone by inhibiting osteoclast differentiation and osteolytic activity. A decrease in bone marrow unsaturated fatty acid might be linked to bone matrix loss. In this study, we observed that the practice of soccer could increase the marrow UI compared with healthy controls; such changes were detected only in the left femoral neck. We believe that physical activity, which increases the proportion of unsaturated fatty acids in the bone marrow, is beneficial to bone integrity. The research data about unsaturated fatty acids of bone marrow after regular and durable high-impact exercise is rather poor and inconsistent.

In contrast to our results, a pilot study reported that the marrow UI was not associated with running performed by adolescents (42), which may be because the mode and intensity of exercise were different in our study. Arsić et al. (43) observed that soccer players have relatively higher monounsaturated fatty acids and lower polyunsaturated fatty acids measurements by gas chromatography than sedentary subjects. This is not entirely consistent with our findings, which may occur because bone marrow fat is a relatively independent fat depot different from the other adipocytes in the body. We speculate that the reason that an increased UI value was only detected in the left femoral neck may be as follows. Firstly, when a soccer player kicks the ball, all of the weight is concentrated on the left lower limb. Different mechanical stimulation causes various changes in the bilateral lower extremities. Secondly, the marrow of femoral neck is predominantly rMAT, and the distal tibia is cMAT, which can explain why the tibial UI remains stable. These findings support the idea that mechanical stimulation with specific regional differences may contribute to disparate outcomes in the UI value. Although, this mechanism warrants further investigation.

Interestingly, we found that both players and healthy controls displayed side-to-side differences in bone marrow unsaturated fatty composition in the femoral neck and distal tibia. The right femoral neck and distal tibia had a higher UI than the left femoral neck in both groups. This may be related to the mechanical stimulus in various structures. No studies on side-to-side differences have been performed in marrow fat conversion caused by mechanical stimulation. Zouch et al. (41) reported that soccer players performing arms muscle strengthening exercises showed a higher $\mathrm{BMD}$ and $\mathrm{BMC}$ in non-dominant arm than the controls after 3-year fellow-up, although they remained similar in dominant arm. All of this implied that side-to-side differences and region-specific variation must be considered in the effect of mechanical stimuli on bone marrow.

There were some limitations in our study that should be noted. Firstly, the cross-sectional study design limits our ability to determine causality. A future longitudinal study is needed to clarify whether soccer practice or other reasons, like long-term diet, cause bone marrow content and composition changes. Secondly, the sample size was small, and stratified analysis of BMI could not be conducted. Thirdly, only young men with or without professional soccer training were analyzed, and thus, the results cannot be applied to women, the elderly, or other modes of exercise. Fourthly, because of the susceptibility effects of the trabecular bone $(44,45)$ and poor resolution of MRS, several small lipid peaks were not shown in all subjects, and their contributions were not taken into account. We attempted to 
calculate the FC and UI using several other fitted fat peaks in the distal tibia; however, the results with more fitted lipid peaks showed no statistical difference compared to our previous choice. These results can be viewed in Table S1. Although several fatty acids contribute to total marrow fat and water content, we believed that our choice of fat peaks could show the overall degree of marrow content, which is consistent with other studies $(13,16)$. Finally, we did not assess the BMD or BMC, which may be more helpful to confirm the relationship between bone marrow fat and bone health in soccer players.

A strength of our study is that it is the first detailed study to measure the bone marrow composition of a variety of specific anatomical regions in healthy professional soccer players using a non-radioactive approach, providing additional insight into the effects of exercise on bone marrow transformation.

Professional soccer training is indeed associated with changes in bone marrow FC and UI, which harness the bone's sensitivity to mechanical signals and regulate the attenuation of marrow fat accrual. The lumbar spine and femoral neck marrow are particularly sensitive to the mechanical loads elicited by soccer practice. Compared to the healthy subjects, the UI of soccer players was significantly higher in the left femoral neck, although the UI values were not notably different in other sites. Overall, soccer practice may be considered an activity that positively affects bone marrow fat by inhibiting adipogenesis.

\section{Acknowledgments}

Funding: The National Natural Science Foundation of China (Grant No. 81801653, 81871349, and 61671228), the Science and Technology Planning Project of Guangdong Province (grant No. 2017B090912006), and the Medical Scientific Research Foundation of Guangdong Province (grant No. A2018268).

\section{Footnote}

Conflicts of Interest: All authors have completed the ICMJE uniform disclosure form (available at http://dx.doi. org/10.21037/qims-21-64). The authors have no conflicts of interest to declare.

Ethical Statement: The authors are accountable for all aspects of the work in ensuring that questions related to the accuracy or integrity of any part of the work are appropriately investigated and resolved. This study was conducted following the Declaration of Helsinki (as revised in 2013) and was approved by the institutional review board of the Third Affiliated Hospital of Southern Medical University. Informed consent was obtained from all individual participants of this study and any accompanying images.

Open Access Statement: This is an Open Access article distributed in accordance with the Creative Commons Attribution-NonCommercial-NoDerivs 4.0 International License (CC BY-NC-ND 4.0), which permits the noncommercial replication and distribution of the article with the strict proviso that no changes or edits are made and the original work is properly cited (including links to both the formal publication through the relevant DOI and the license). See: https://creativecommons.org/licenses/by-nc-nd/4.0/.

\section{References}

1. Zhao Y, Huang M, Ding J, Zhang X, Spuhler K, Hu S, Li M, Fan W, Chen L, Zhang X, Li S, Zhou Q, Huang C. Prediction of Abnormal Bone Density and Osteoporosis From Lumbar Spine MR Using Modified Dixon Quant in 257 Subjects With Quantitative Computed Tomography as Reference. J Magn Reson Imaging 2019;49:390-9.

2. Aoki T, Yamaguchi S, Kinoshita S, Hayashida Y, Korogi Y. Quantification of bone marrow fat content using iterative decomposition of water and fat with echo asymmetry and least-squares estimation (IDEAL): reproducibility, site variation and correlation with age and menopause. $\mathrm{Br} \mathrm{J}$ Radiol 2016;89:20150538.

3. Bertheau RC, Lorbeer R, Nattenmüller J, Wintermeyer E, Machann J, Linkohr B, Peters A, Bamberg F, Schlett CL. Bone marrow fat fraction assessment in regard to physical activity: KORA FF4-3-T MR imaging in a populationbased cohort. Eur Radiol 2020;30:3417-28.

4. Hagman M, Helge EW, Hornstrup T, Fristrup B, Nielsen JJ, Jørgensen NR, Andersen JL, Helge JW, Krustrup P. Bone mineral density in lifelong trained male football players compared with young and elderly untrained men. J Sport Health Sci 2018;7:159-68.

5. Lozano-Berges G, Matute-Llorente A, GonzalezAguero A, Gomez-Bruton A, Gomez-Cabello A, VicenteRodriguez G, Casajus JA. Soccer helps build strong bones during growth: a systematic review and meta-analysis. Eur J Pediatr 2018;177:295-310.

6. Calbet JA, Dorado C, Díaz-Herrera P, Rodríguez- 
Rodríguez LP. High femoral bone mineral content and density in male football (soccer) players. Med Sci Sports Exerc 2001;33:1682-7.

7. Uzunca K, Birtane M, Durmus-Altun G, Ustun F. High bone mineral density in loaded skeletal regions of former professional football (soccer) players: what is the effect of time after active career? Br J Sports Med 2005;39:154-7; discussion 157.

8. Ambrosi TH, Schulz TJ. The emerging role of bone marrow adipose tissue in bone health and dysfunction. J Mol Med (Berl) 2017;95:1291-301.

9. Napoli N, Chandran M, Pierroz DD, Abrahamsen B, Schwartz AV, Ferrari SL; IOF Bone and Diabetes Working Group. Mechanisms of diabetes mellitus-induced bone fragility. Nat Rev Endocrinol 2017;13:208-19.

10. Horowitz MC, Berry R, Holtrup B, Sebo Z, Nelson T, Fretz JA, Lindskog D, Kaplan JL, Ables G, Rodeheffer MS, Rosen CJ. Bone marrow adipocytes. Adipocyte 2017;6:193-204.

11. Hardouin P, Rharass T, Lucas S. Bone marrow adipose tissue: to be or not to be a typical adipose tissue? Front Endocrinol (Lausanne) 2016;7:85.

12. Pino AM, Rodriguez JP. Is fatty acid composition of human bone marrow significant to bone health? Bone 2019;118:53-61.

13. Yeung DK, Griffith JF, Antonio GE, Lee FK, Woo J, Leung PC. Osteoporosis is associated with increased marrow fat content and decreased marrow fat unsaturation: a proton MR spectroscopy study. J Magn Reson Imaging 2005;22:279-85.

14. Yu EW, Greenblatt L, Eajazi A, Torriani M, Bredella MA. Marrow adipose tissue composition in adults with morbid obesity. Bone 2017;97:38-42.

15. Bredella MA, Torriani M, Ghomi RH, Thomas BJ, Brick DJ, Gerweck AV, Rosen CJ, Klibanski A, Miller KK. Vertebral Bone Marrow Fat Is Positively Associated With Visceral Fat and Inversely Associated With IGF-1 in Obese Women. Obesity 2011;19:49-53.

16. Patsch JM, Li X, Baum T, Yap SP, Karampinos DC, Schwartz AV, Link TM. Bone marrow fat composition as a novel imaging biomarker in postmenopausal women with prevalent fragility fractures. J Bone Miner Res 2013;28:1721-8.

17. Baum T, Yap SP, Karampinos DC, Nardo L, Kuo D, Burghardt AJ, Masharani UB, Schwartz AV, Li X, Link TM. Does vertebral bone marrow fat content correlate with abdominal adipose tissue, lumbar spine bone mineral density, and blood biomarkers in women with type 2 diabetes mellitus? J Magn Reson Imaging 2012;35:117-24.

18. Wang L, Salibi N, Chang G, Vieira RL, Babb JS, Krasnokutsky S, Abramson S, Regatte RR. Assessment of subchondral bone marrow lipids in healthy controls and mild osteoarthritis patients at $3 \mathrm{~T}$. NMR Biomed 2012;25:545-55.

19. Peterson P, Trinh L, Mansson S. Quantitative (1) H MRI and MRS of fatty acid composition. Magn Reson Med 2021;85:49-67.

20. Karampinos DC, Ruschke S, Dieckmeyer M, Diefenbach M, Franz D, Gersing AS, Krug R, Baum T. Quantitative MRI and spectroscopy of bone marrow. J Magn Reson Imaging 2018;47:332-53.

21. Xu K, Sigurdsson S, Gudnason V, Hue T, Schwartz A, Li X. Reliable quantification of marrow fat content and unsaturation level using in vivo MR spectroscopy. Magn Reson Med 2018;79:1722-9.

22. Naressi A, Couturier C, Castang I, de Beer R, GraveronDemilly D. Java-based graphical user interface for MRUI, a software package for quantitation of in vivo/medical magnetic resonance spectroscopy signals. Comput Biol Med 2001;31:269-86.

23. Naressi A, Couturier C, Devos JM, Janssen M, Mangeat C, de Beer R, Graveron-Demilly D. Java-based graphical user interface for the MRUI quantitation package. MAGMA 2001;12:141-52.

24. Stefan D, Di Cesare F, Andrasescu A, Popa E, Lazariev A, Vescovo E, Strbak O, Williams S, Starcuk Z, Cabanas M. Quantitation of magnetic resonance spectroscopy signals: the jMRUI software package. Meas Sci Technol 2009;20:104035.

25. Weis J, Johansson L, Ortiz-Nieto F, Ahlstrom H. Assessment of lipids in skeletal muscle by LCModel and AMARES. J Magn Reson Imaging 2009;30:1124-9.

26. Vanhamme L, van den Boogaart A, Van Huffel S. Improved method for accurate and efficient quantification of MRS data with use of prior knowledge. J Magn Reson 1997;129:35-43.

27. Loffroy R, Terriat B, Jooste V, Robin I, Brindisi MC, Hillon P, Verges B, Cercueil JP, Petit JM. Liver fat content is negatively associated with atherosclerotic carotid plaque in type 2 diabetic patients. Quant Imaging Med Surg 2015;5:792-8.

28. Bredella MA, Fazeli PK, Daley SM, Miller KK, Rosen CJ, Klibanski A, Torriani M. Marrow fat composition in anorexia nervosa. Bone 2014;66:199-204.

29. Schellinger D, Lin CS, Hatipoglu HG, Fertikh D. Potential value of vertebral proton MR spectroscopy in 
determining bone weakness. AJNR Am J Neuroradiol 2001;22:1620-7.

30. Belavy DL, Quittner MJ, Ridgers ND, Shiekh A, Rantalainen T, Trudel G. Specific Modulation of Vertebral Marrow Adipose Tissue by Physical Activity. J Bone Miner Res 2018;33:651-7.

31. Sen B, Xie Z, Case N, Ma M, Rubin C, Rubin J. Mechanical strain inhibits adipogenesis in mesenchymal stem cells by stimulating a durable beta-catenin signal. Endocrinology 2008;149:6065-75.

32. Yuki A, Yotani K, Tamaki H, Kasuga N, Takekura H. Upregulation of osteogenic factors induced by highimpact jumping suppresses adipogenesis in marrow but not adipogenic transcription factors in rat tibiae. Eur J Appl Physiol 2010;109:641-50.

33. Pagnotti GM, Styner M, Uzer G, Patel VS, Wright LE, Ness KK, Guise TA, Rubin J, Rubin CT. Combating osteoporosis and obesity with exercise: leveraging cell mechanosensitivity. Nat Rev Endocrinol 2019;15:339-55.

34. Weinreb JC. MR Imaging of Bone Marrow: A Map Could help. Radiology 1990;177:23-4.

35. Shellock FG, Morris E, Deutsch AL, Mink JH, Kerr R, Boden SD. Hematopoietic bone marrow hyperplasia: high prevalence on MR images of the knee in asymptomatic marathon runners. AJR Am J Roentgenol 1992;158:335-8.

36. Gurevitch O, Slavin S, Feldman AG. Conversion of red bone marrow into yellow - Cause and mechanisms. Med Hypotheses 2007;69:531-6.

37. Devlin MJ, Rosen CJ. The bone-fat interface: basic and clinical implications of marrow adiposity. Lancet Diabetes Endocrinol 2015;3:141-7.

38. Scheller EL, Doucette CR, Learman BS, Cawthorn WP, Khandaker S, Schell B, Wu B, Ding SY, Bredella MA, Fazeli PK, Khoury B, Jepsen KJ, Pilch PF, Klibanski A, Rosen CJ, MacDougald OA. Region-specific variation

Cite this article as: Wang J, Yi P, Huang Y, Yu Q, Mei Y, Chen J, Feng Y, Zhang X. Quantitative evaluation of bone marrow fat content and unsaturated fatty index in young male soccer players using proton magnetic resonance spectroscopy $\left({ }^{1} \mathrm{H}-\mathrm{MRS}\right)$ : a preliminary study. Quant Imaging Med Surg 2021;11(10):4275-4286. doi: 10.21037/qims-21-64 in the properties of skeletal adipocytes reveals regulated and constitutive marrow adipose tissues. Nat Commun 2015;6:7808.

39. Singhal V, Torre Flores LP, Stanford FC, Toth AT, Carmine B, Misra M, Bredella MA. Differential associations between appendicular and axial marrow adipose tissue with bone microarchitecture in adolescents and young adults with obesity. Bone 2018;116:203-6.

40. Söderman K, Bergstrom E, Lorentzon R, Alfredson H. Bone mass and muscle strength in young female soccer players. Calcif Tissue Int 2000;67:297-303.

41. Zouch M, Zribi A, Alexandre C, Chaari H, Frere D, Tabka $Z$, Vico L. Soccer increases bone mass in prepubescent boys during growth: a 3-yr longitudinal study. J Clin Densitom 2015;18:179-86.

42. Huovinen V, Viljakainen H, Hakkarainen A, Saukkonen T, Toiviainen-Salo S, Lundbom N, Lundbom J, Makitie $\mathrm{O}$. Bone marrow fat unsaturation in young adults is not affected by present or childhood obesity, but increases with age: A pilot study. Metabolism 2015;64:1574-81.

43. Arsić A, Vučić V, Tepšić J, Mazić S, Djelić M, Glibetić M. Altered plasma and erythrocyte phospholipid fatty acid profile in elite female water polo and football players. Appl Physiol Nutr Metab $2012 ; 37: 40-7$.

44. Lundbom J, Bierwagen A, Bodis K, Apostolopoulou M, Szendroedi J, Müssig K, Hwang JH, Roden M. ${ }^{1} \mathrm{H}-\mathrm{MRS}$ of femoral red and yellow bone marrow fat composition and water content in healthy young men and women at $3 \mathrm{~T}$. MAGMA 2019;32:591-7.

45. Cordes C, Baum T, Dieckmeyer M, Ruschke S, Diefenbach MN, Hauner H, Kirschke JS, Karampinos DC. MR-Based Assessment of Bone Marrow Fat in Osteoporosis, Diabetes, and Obesity. Front Endocrinol (Lausanne) 2016;7:74. 


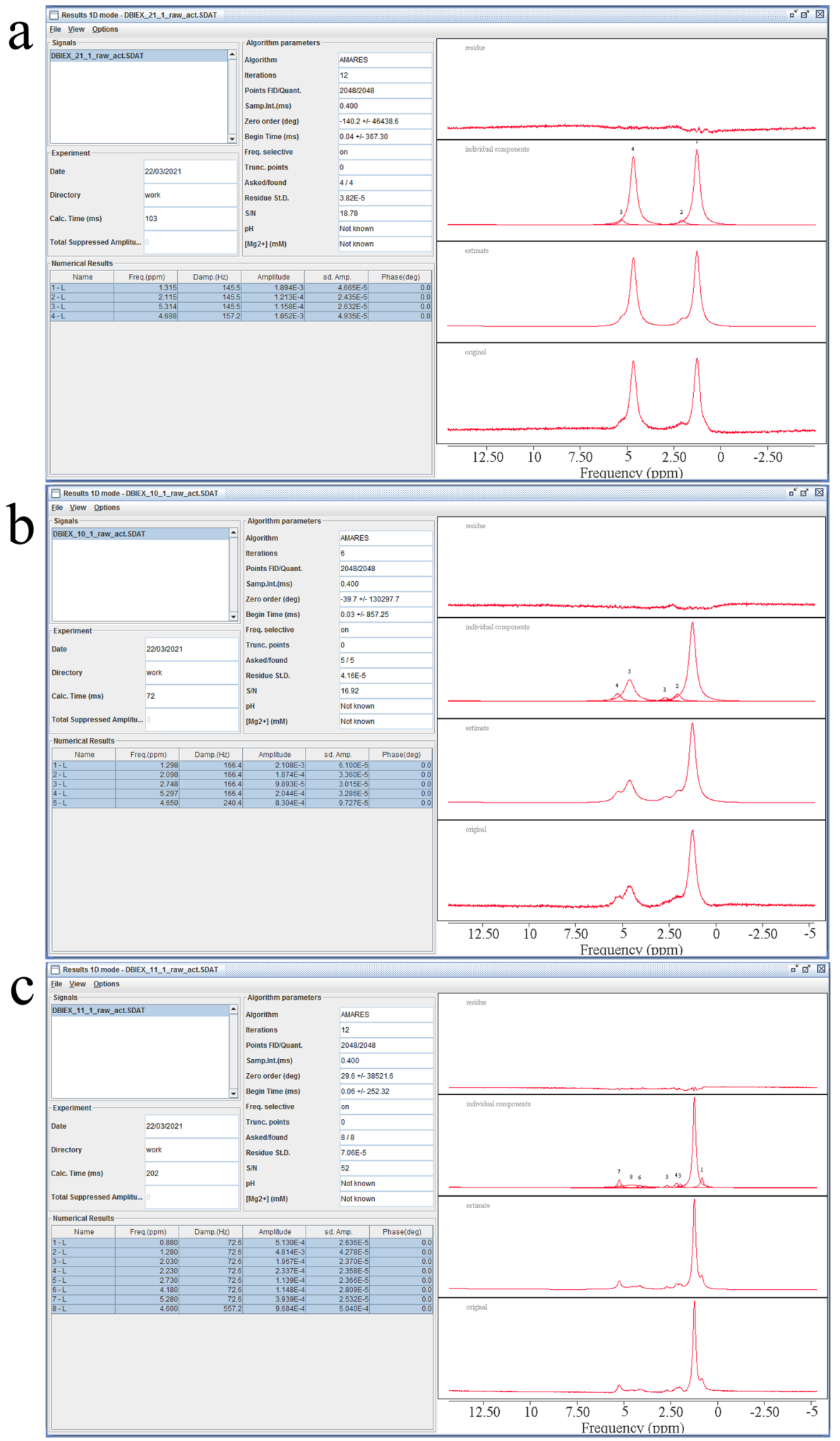

Figure S1 Fitting results of the corresponding spectrum in the vertebral body (A), femoral neck (B), and distal tibia (C), including the residue, individual components, as well as the estimated and original spectrum. 
Table S1 The mean \pm standard deviation of fat content and unsaturated fatty index values of bilateral tibias between the players and control groups with fitting all lipid peak

\begin{tabular}{lccccc}
\hline \multirow{2}{*}{ Location } & \multicolumn{3}{c}{ Fat content $(\%)$} & \multicolumn{3}{c}{ Unsaturated fatty index (\%) } \\
\cline { 2 - 6 } & Players & Controls & P value $^{\mathrm{a}}$ & Players & Controls $^{\mathrm{P} \text { value }}$ \\
\hline Left tibia & $90.78 \pm 3.84$ & $92.77 \pm 2.07$ & 0.052 & $6.69 \pm 1.16$ & $7.23 \pm 0.82$ \\
Right tibia & $92.03 \pm 2.23$ & $93.22 \pm 2.26$ & 0.104 & $8.91 \pm 5.00$ & $8.03 \pm 1.08$ \\
\hline
\end{tabular}

Values are expressed as mean \pm standard deviation. ${ }^{\text {, }}$, calculated using the independent samples t-test to show the difference between soccer players and healthy subjects. 